\title{
A Method for Extracting a QRS Wave in an ECG Based on DP Matching
}

\author{
Member Yukinori Suzuki (Muroran Institute of Technology) \\ Non-member Yukihisa Kurimoto (Muroran Institute of Technology) \\ Non-member Sajjad Mohsin (Muroran Institute of Technology) \\ Non-member Junji Maeda (Muroran Institute of Technology)
}

\begin{abstract}
We propose a method for extracting QRS waves in an electrocardiogram (ECG) based on DP matching using an MIT/BIH arrhythmia database. The QRS wave is extracted to detect the characteristic points (CPs), the $\mathrm{Q}$ and $\mathrm{S}$ points, in an ECG. In the proposed method, a preprocessor, DP matching, and a detection algorithm are used. The preprocessor is used to divide an ECG into individual cardiac cycles. DP matching is used for selecting a template pattern. The detection algorithm is used to find CPs from the selected template pattern. The results of an experiment showed that SDs of detection errors using our method are 5.1 and 1.9 for the detection of the $Q$ and $S$ point, respectively. These small SDs indicate the reliability of our method.
\end{abstract}

Keywords: electrocardiogram (ECG), QRS wave, DP matching

\section{Introduction}

An electrocardiogram (ECG) is a recording that enables easy and noninvasive measurement of the electrical potential of the heart. ECGs are often used to diagnose heart diseases. A typical ECG is illustrated in Fig. 1. To assist physicians in the tedious routines of analyzing an ECG, computerized ECG analyzers have been developed. The most popular ECG analyzer is a beat-to-beat analyzer. However, waves in the ECG have to be analyzed automatically for precise diagnosis. The QRS wave is the most remarkable wave and is therefore used as a reference wave for detection of other waves. The QRS wave can be extracted by detection of two characteristic points (CPs), the $\mathrm{Q}$ and $\mathrm{S}$ points, in an ECG. In this paper, we propose a method based on DP matching for detecting the $\mathrm{Q}$ and $\mathrm{S}$ points in order to extract the QRS wave. This method is developed using an MIT/BIH arrhythmia database.

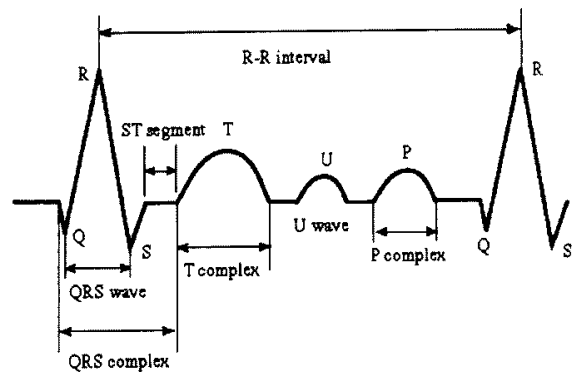

Fig. 1. A cardiac cycle of an ECG. The ECG consists of complexes and waves.

2. Detection of CPs based on DP matching ECGs in the MIT/BIH arrhythmia database are digitized at a rate of $360 \mathrm{~Hz}$. This rate is not sufficient for precise detection of CPs. A preprocessor is therefore used to linearly interpolate two successive samples of an ECG. The $\mathbf{R}$ points are detected in order to divide the $\mathrm{ECG}$ into individual cardiac cycles. Then the part of the ECG $100 \mathrm{~ms}$ in length from the $\mathrm{R}$ point towards the $\mathrm{P}$ point (the $\mathrm{QR}$ part) is extracted for $\mathrm{Q}$ point detection. For $\mathrm{S}$ point detection, the RS part is also extracted in the same manner.

Dynamic programming (DP), originally proposed by Bellman, provides an efficient mechanism for sequential decision-making. The mathematical basis of dynamic programming is the principle of optimality. DP matching is a method for pattern recognition by DP.

We prepared two template patterns for DP matching, as shown in Fig. 2. These patterns are specified empirically by observing the large number of QRS waves $^{(1)}$ For $\mathrm{Q}$ point detection, the $\mathrm{QR}$ part is compared with template patterns as a reference pattern. Since the ECG is digitized at a rate of $720 \mathrm{~Hz}$, there are 72 samples in the QR part. Each template pattern also consists of 72 samples. The distances between the reference pattern and the two template patterns are computed by DP matching. The time series of the template and reference patterns are $\{T(1), T(2), \ldots, T(J)\}$ and $\{R(1), R(2), \ldots, R(I)\}$, respectively. Both $J$ and $I$ are $72 . d(i, j)$ stands for the distance between $T(i)$ and $R(j) . g(i, j)$ stands for the distance between the two series $\{T(1), T(2), \ldots, T(j)\}$ and $\{R(1), R(2), \ldots, R(j)\}$ which are computed by an algorithm of DP matching. Computation is carried out using by the following algorithm ${ }^{(2)}$

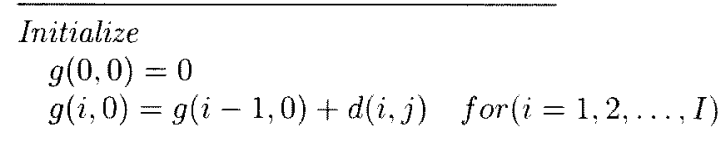




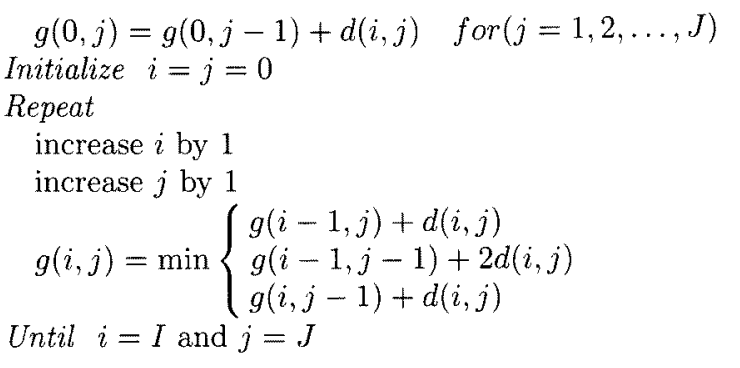

The distance between the template pattern (a) and the $\mathrm{QR}$ part is computed using the above. The distance between the template pattern (b) and the $Q R$ part is also computed. The template pattern showing less $g(I, J)$ is employed to detect the Q point.

The template pattern chosen is stretched and/or shrunk by the algorithm of DP matching, and is represented by $\tilde{Q R}$. The $\mathrm{Q}$ point is detected in $\tilde{Q R}$ based on a slope detection technique using the following algorithm. The $\mathrm{S}$ point is detected in the same manner.

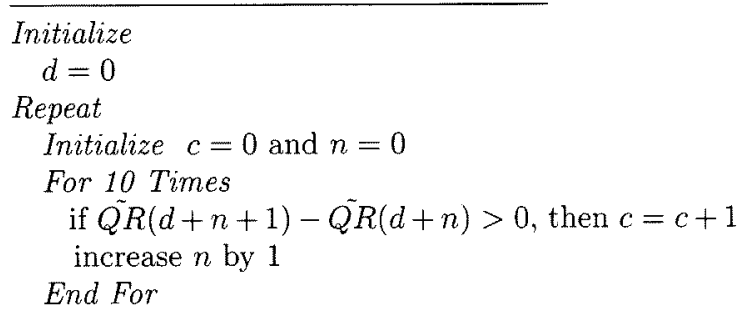

if $c \geq 7$, then $d$ is the Q point, and algorithm ends. increase $d$ by 1

Until $d \leq 72$

\section{Results and Discussion}

Detection of CPs by the proposed method was evaluated using the MIT/BIH arrhythmia database. We chose record numbers of ECGs in which we could visually locate the CPs clearly. The ECG record numbers chosen were $100,103,112,113,115,116,117,122,123$, and 124. A total of 2,000 cardiac cycles of ECGs were used for evaluation. For the evaluation, the $\mathrm{Q}$ point was defined as the first visually remarkable inflecting part of ECG from the $R$ point towards the $P$ point. The S point was also defined as the first visually remarkable inflecting part of the ECG from the $\mathrm{R}$ point towards the $\mathrm{T}$ point. To evaluate detected CPs, a part of the ECG including the QRS wave was expanded and drawn on the display of a personal computer. A referee visually determined the locations of the CPS both by examining the slope change of the ECG and the wave form of the ECG before and after visual detection of the CPs. The $\mathrm{CPs}$ located by the referee were considered to be the true CPs.

Detection errors were computed as

$$
S_{\text {error }}=\left|n_{c}-n_{v}\right|
$$

where $n_{c}$ is the point on the ECG where a CP was detected using our method, and $n_{v}$ is the point on the ECG where a CP was located by the referee. Fig. 2 shows a summary of the results of evaluation. For $Q$

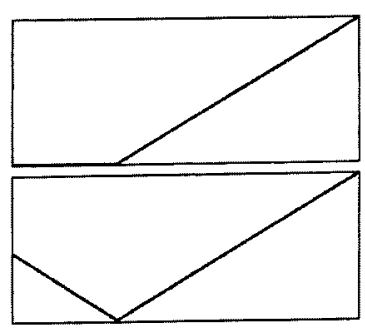

Fig. 2. Template patterns for DP matching. The upper pattern is template pattern (a) and the lower is template pattern (b)
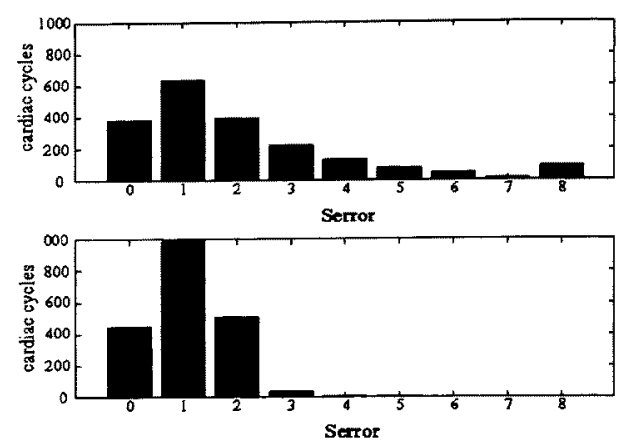

Fig. 3. Summary of the errors for detection of $Q$ and $\mathrm{S}$ points detections.

point detection, the rate of accuracy with $S_{\text {error }} \leq 5.0$ was $92.1 \%$, and the rate of accuracy with $S_{\text {error }} \leq 5.0$ was $99.7 \%$ for $\mathrm{S}$ point detection. The error in $\mathrm{Q}$ point detection is larger. The CSE committee has shown the limit of the SD for the detection error using a CSE ECG library, which is a different ECG database from the MIT/BIH arrhythmia database ${ }^{(3)}$ It can be consider that we can evaluate our method by the SDs for the detection error determined by the CSE committee, because ECGs in the MIT/BIH database are abnormal, which could provide severe condition for the evaluation. The limit of the SDs by the CSE committee are 6.5 and 11.6 for detection of $\mathrm{Q}$ and $\mathrm{S}$ points, respectively. Since the SDs in our method are 5.1 and 1.9 for the $\mathrm{Q}$ and $\mathrm{S}$ points detections, respectively, The SDs show the reliability of our method. Moreover, the proposed method is based on a simple algorithm, and a small computational burden enables real-time processing. The proposed method could therefore be used for real-time monitoring of patients in a coronary care unit and for monitoring of patients living at home.

(Manuscript received January 26, 2001)

\section{References}

(1) A study on an automatic ECG analyzing system, M.S. Thesis, Muroran Institute of Technology, 1997.

(2) S. Nakagawa, Pattern Information Processing (in Japanese), Tokyo: Maruzen, 1992.

(3) The CSE working party, Recommendation for measurement of standards in electrocardiography, European Heart Journal, vol. 6, pp.815-825, 1985 